\section{Recommandations pour le suivi des néoplasmes colorectaux (adénome, carcinome)}

\author{
SPGES et GastroMéd Suisse
}

\section{Fondements}

La Société Professionnelle des Gastro-Entérologues FMH Suisses (SPGES) publie ces recommandations révisées avec la collaboration de GastroMed Suisse (GMS). Elles remplacent la première version de 1996 et ont pour objet de garantir la qualité.

Ces recommandations sont fondées sur des directives qui existent déjà dans d'autres pays et sur la littérature actuelle (voir références). Pour ce qui est des polypes ou adénomes, la base de données est fiable, alors que pour les carcinomes il demeure des incertitudes. C'est pourquoi ces recommandations ont à nouveau été revues par une conférence de consensus, à laquelle ont participé des représentants des sociétés professionnelles et associations importantes dans ce domaine (voir ci-dessous). D’autres révisions sont prévues.

\section{Interprétation}

En publiant ces recommandations, la SPGES est parfaitement consciente du fait qu'il est difficile de trouver une voie acceptable entre les exigences de la politique de santé, d'une part, et les revendications en matière de liberté de l'exercice professionnel, d'autre part. Il faut donc considérer ces recommandations comme un cadre pour le suivi rationnel de ces affections en question. Elles représentent un compromis, surtout pour le carcinome colorectal. Il est possible de s'écarter du schéma recommandé. De plus, le degré de gravité des maladies existantes et l'espérance de vie déterminent aussi les modalités du suivi.
Liste des invités à la conférence de consensus

(du 18 novembre 1998)

- Membres du comité de la SPGES;

- Chefs et cheffes des centres de formation en gastro-entérologie A et B;

- Société Suisse d'Oncologie/Hématologie;

- Société Suisse de Chirurgie Viscérale;

- Société Suisse de Radiologie Diagnostique;

- Société Suisse de Pathologie;

- Société Suisse de Médecine Interne;

- Société Suisse de Médecine Générale;

- Ligue Suisse contre le Cancer;

- Registre Suisse du Cancer;

- Organisation de patients;

\section{Suivi après polypectomie colo-rectale}

1. Adénome avec carcinome*

(sous réserve d'une résection curative ${ }^{* *}$ )

Au moins un contrôle endoscopique du site de résection dans les 12 mois suivant l'opération et nouvelle coloscopie complète après 5 et 10 ans.

* Critère de l'O.M.S. de 1989: franchissement de la muscularis mucosae.

** Critères internationaux (littérature): tumeur bien différenciée ou moyennement différenciée, pas de pénétration dans les vaisseaux lymphatiques ou sanguins, limite de résection à $3 \mathrm{~mm}$ au minimum de la lésion.

2. Adénome caractérisé par au moins l'un des critères suivants

- Diamètre $>=1 \mathrm{~cm}$;

- Nombre $>=3$;

- Villeux ou tubulo-villeux à l'examen histologique;

- Dysplasie sévère.

Coloscopie complète après 5 et 10 ans.

3. Adénomes tubulaires de diamètre inférieur à $1 \mathrm{~cm}$ (nombre: 1 à 2) ou polypes hyperplasiques (quels qu'en soient les dimensions et le nombre) Pas de suivi.

\section{Références}

- Ann Intern Med 1990;113:760-3.

- Gut 1992;21:853-62.

- N Engl J Med 1992;326:658-62.

- N Engl J Med 1993;329:1977-81.

- Ann Intern Med 1993;119:836-43.

- Scand J Gastroenterol 1993;26:239-43.

- Gastrointest Endosc 1993;39:481-5.

- N Engl J Med 1993;328:901-6.

- Dig Dis Sci 1994;39:2282-4.

- Gastroenterology 1994;106:1501-4.

- Scand J Gastroenterol 1995;30:686-92.

- Scand J Gastroenterol 1996;31:834-6.

- Scand J Gastroenterol 1996;31:1006-10.

- Gut 1998;39:449-56.

- ASGE 1998;6:1-4.

- Gut 1998;43:669-74. 


\section{Suivi du carcinome colorectal après opération curative}

Condition préliminaire: coloscopie complète avant ou 3 à 6 mois après l'opération.

\section{Coloscopie}

Tous les 5 ans (néoplasie secondaire?).

\section{Rectosigmoïdoscopie et écho-endoscopie}

(si disponible)

Tous les 6 mois pendant les deux premières années suivant l'opération (récidive loco-régionale après un carcinome du rectum ou du sigmoïde distal?).

\section{Echographie hépatique}

Tous les ans pendant les trois premières années après l'opération.

4. Examens dont l'intérêt n'est pas prouvé

- Bilan biologique;

- CEA;

- Radiographie thoracique;

- CT-scanner.

\section{Références}

- Dis Colon Rectum 1994;37:568-72.

- Ann Surg 1994;219:174-82.

- Endoscopy 1994;26:523-7.

- Ann Surg 1994;220:206-11.

- Dis Colon Rectum 1995;38:619-26.

- Arch Surg 1995;130:1062-7.

- Dis Colon Rectum 1996;39:252-6.

- Am J Gastroenterol 1996;91:2096-8.

- Br J Cancer 1997;75:914-20.

- Dis Colon Rectum 1997;40:15-24.

- Br J Surg 1997;84:666-9.

- Oncology 1997;54:287-92.

- J Chir (Paris) 1997;134:45-50.

- Ann Intern Med 1998;129:27-35.

- Ann Surg 1998;228:59-63.

- Dis Colon Rectum 1998;41:1127-33.

- Dis Colon Rectum 1998;41:1116-26.

- Gastroenterology 1998;114:7-14. 